\title{
Stress relaxation and estimation of activation volume in a commercial hard PZT piezoelectric ceramic
}

\author{
B L CHENG and M J REECE* \\ Department of Materials, Queen Mary and Westfield College, Mile End Road, E1 4NS, London, UK
}

\begin{abstract}
The stress relaxation of ferroelectric/piezoelectric material was studied using compression testing. The deformation was produced by the switching of ferroelectric domains. The internal stresses were estimated by decremental stress relaxation during unloading. The results were interpreted in terms of reversible and irreversible switching of the domains.
\end{abstract}

Keywords. Ferroelectric/piezoelectric material; deformation; decremental stress relaxation; domains.

\section{Introduction}

The $\mathrm{Pb}(\mathrm{Zr}, \mathrm{Ti}) \mathrm{O}_{3}(\mathrm{PZT})$ ceramics are widely used for the sensors and actuators. The long-term performance of the devices depends on the stability of the materials under service conditions. The electrical and mechanical cycling of piezoelectrics can cause irreversible microstructural damage in the form of micro-cracking and reversible damage of the poled ferroelectric domain structure. The problem of degradation is becoming more serious as components are increasingly being used in applications that subject them to relatively large cyclic, electrical and mechanical loadings. Even relatively low stress may produce reversible and irreversible domain reorientation (> $50 \mathrm{MPa}$ ) (Calderon Moreno et al 1999).

The study of stress-strain hysteresis in compression has been used to specify the stress levels at which reversible and irreversible processes take place (Calderon Moreno et al 1999). In this work we have focused on the stress relaxation produced by ferroelectric domain switching in order to determine the kinetics of switching. Furthermore, a decremental stress relaxation during unloading was used to estimate internal stresses.

\section{Materials and experimental}

A commercial $\mathrm{Pb}(\mathrm{Zr}, \mathrm{Ti}) \mathrm{O}_{3}$ material provided by Morgan Matroc, Transducer Division, Southampton, UK, of 4D type (piezoelectric 'hard' with $d_{33}=315 \mathrm{pC} / \mathrm{N}$ ) was studied. The nominal dimensions of the compression specimens were $2 \times 2 \times 4 \mathrm{~mm}$, with poling direction along the $4 \mathrm{~mm}$ length. Compression testing was performed in an Instron testing machine 8511 with a self aligning jig (Calderon Moreno et al 1999). The testing was carried out

\footnotetext{
*Author for correspondence
}

in displacement control for stress relaxation testing at a strain rate of $3 \% / \mathrm{min}$. An active capacitor transducer measured the displacement of specimen with a sensitivity of $367 \mu \mathrm{m} / \mathrm{V}$.

\section{Results and discussion}

Figure 1 shows stress-strain curves for the PZT 4D stressed up to $400 \mathrm{MPa}$. The results show three stages for the loading run: (i) a linear region when the stress is less than about $50 \mathrm{MPa}$; (ii) an easy deformation region when the stress is in between 50 and $300 \mathrm{MPa}$ and (iii) a hardening region at high stress due to exhaustion of the available non $-180^{\circ}$ domains that could be switched. On unloading there is a large recovery of strain due to the effect of internal stresses.

\subsection{Estimation of internal stress}

Figure 2 shows stress relaxation curves during unloading at different stress levels for the PZT 4D ceramic. The internal stress was estimated by using the incremental unloading technique developed by MacEwen et al (1969). In this technique the stress relaxation over a finite time interval is measured at several stress levels. When the applied stress level is higher than the internal stress, normal relaxation behaviour is observed, but when the applied stress level is below the internal stress the relaxation is negative. In other words, the stress level at which no relaxation is observed corresponds to the internal stress. The early application of the technique was carried out by Esaklul et al (1980) on unpoled PZT. Figure 3 shows a linear relationship between the peak-applied stress and the estimated internal stress for the PZT 4D ceramic. The result shows that the internal stress is about $70 \%$ of the maximum applied stress. 


\subsection{Estimation of apparent activation volume}

Figure 4 shows the stress relaxation of the PZT ceramic for incrementally increased levels of stress over a time interval of $60 \mathrm{sec}$. As a first approximation, such stress relaxation curves were analysed by fitting to the following equation (Guiu and Pratt 1964)

$$
\Delta \sigma=-k T / V_{\mathrm{a}} \ln \left(1+t / \tau_{\mathrm{c}}\right),
$$

where $k$ is Boltzman's constant, $T$ the temperature in $\mathrm{K}$, $V_{\mathrm{a}}$ an apparent activation volume and $\tau_{\mathrm{c}}$ the relaxation time.

Figure 5 shows an example of a stress relaxation curve and the corresponding fitting curve. In this way, an appa-

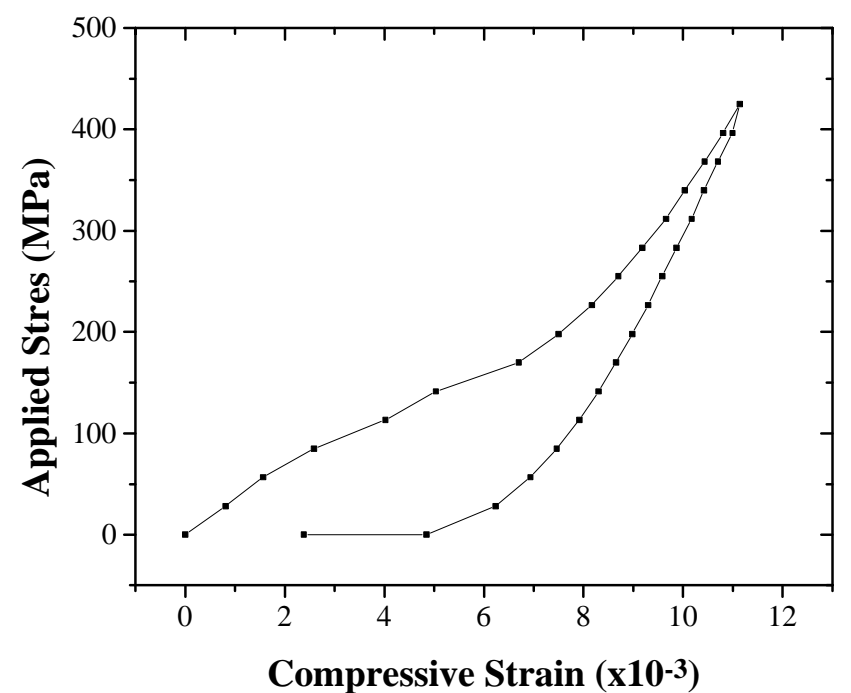

Figure 1. Compression stress-strain for hard PZT 4D material parallel to poling. The tests were performed in load control.

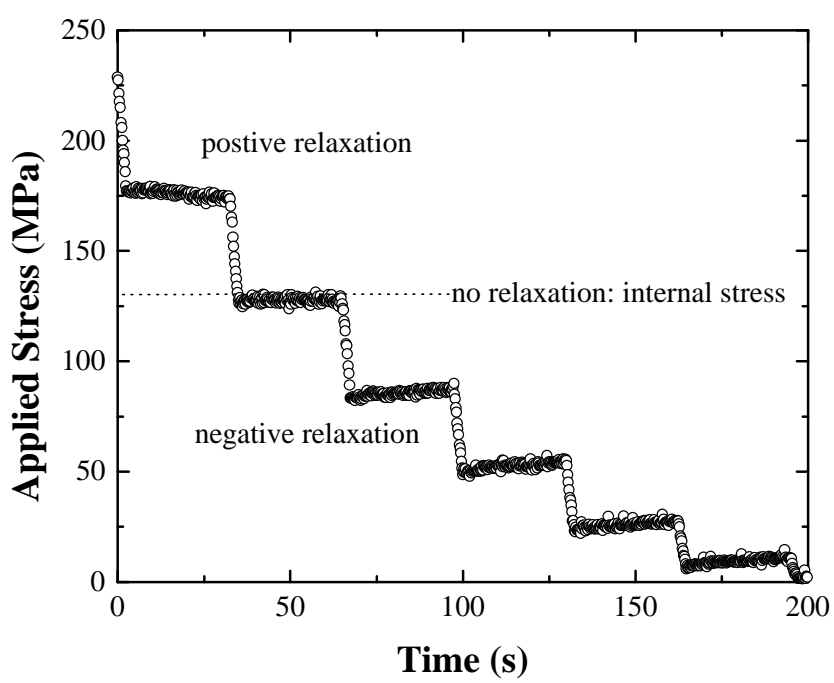

Figure 2. Typical stress relaxation curves for PZT 4D ceramic produced by incremental unloading technique for internal stress estimation. rent activation volume $\left(V_{\mathrm{a}}\right)$ as a function of applied stress was obtained (figure 6). The results show that the activation volume is about $10 \mathrm{~nm}^{3}$ at low applied stresses, and decreases with increasing applied stress. This behaviour can be explained by the exhaustion of the deformation process as the availability of switchable ferroelectric domains is reduced with applied stress. Considering the unit cell volume of the PZT ceramic (about $0.04 \mathrm{~nm}^{3}$ ), the apparent activation volume at low stress is equivalent to about 100 unit cells. This means the switching of ferroelectric domain is a cooperative process involving the shear deformation of many unit cells. When increasing applied stresses, the apparent activation volume is about several tens of unit cells, and the activation volume becomes stable. The estimated values of the activation

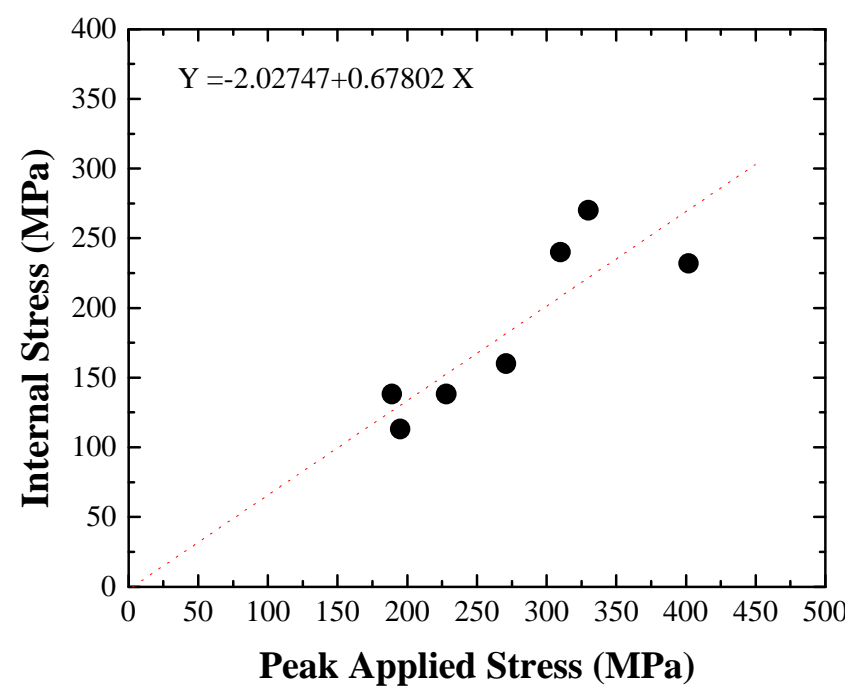

Figure 3. Relationship between peak applied stress and internal stress in PZT 4D ceramics.

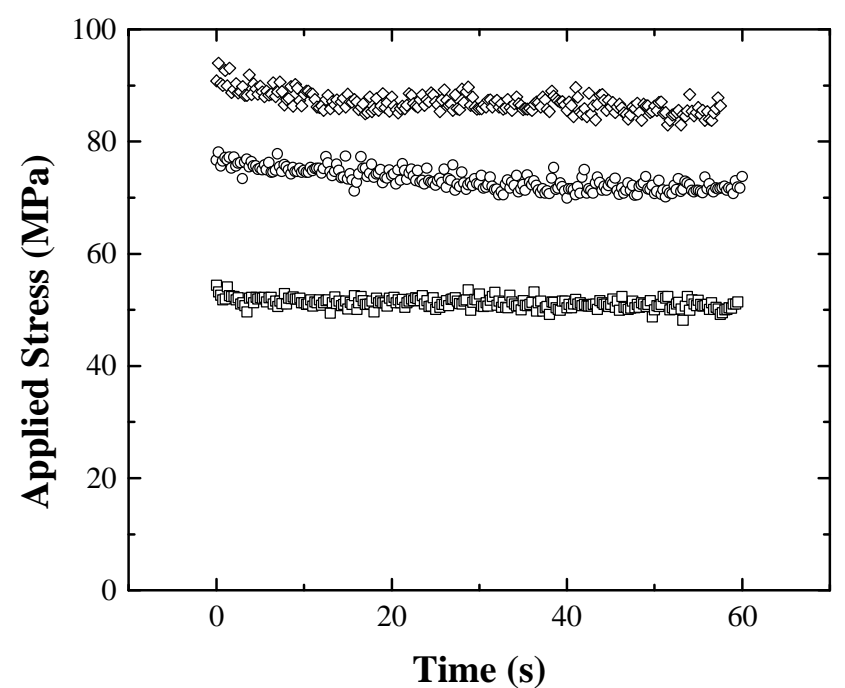

Figure 4. Stress relaxation of PZT 4D ceramic at different levels of applied stress. 


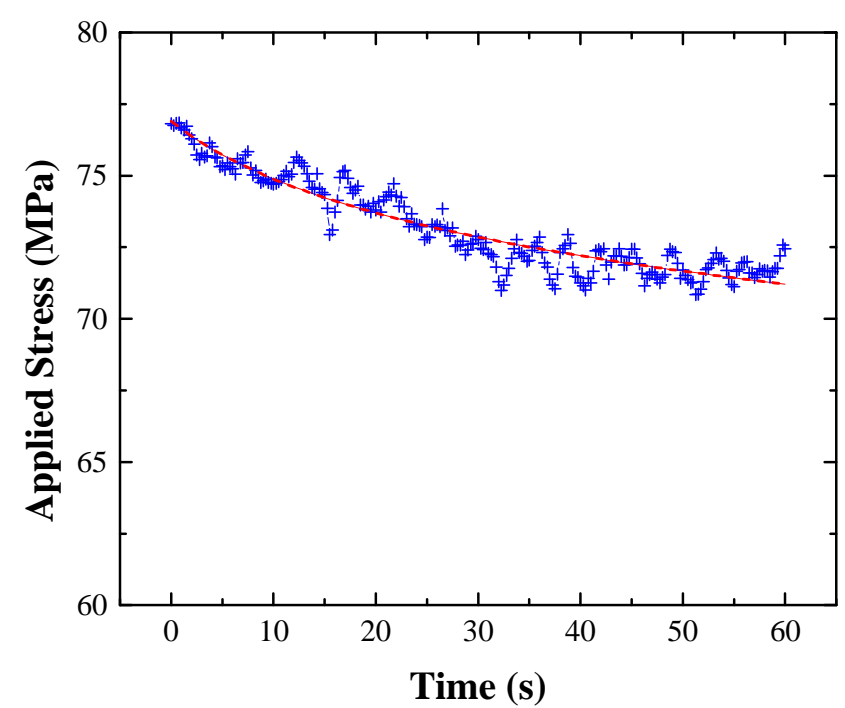

Figure 5. Stress relaxation fitting by logarithmic time dependence.

volumes are similar to that obtained by Esaklul et al (1980).

\section{Conclusions}

The observed large strain of PZT ceramic during compression testing is due to the switching of ferroelectric domains. The internal stresses that develop during deformation are about $70 \%$ of the maximum applied stress. An apparent activation volume was estimated using logarithmic relationships for both the stress relaxations at different stresses. The value of apparent activation volume is about several tens of the unit cell for PZT material. This signifies a cooperative behaviour of the domain switching during compression of poled materials.

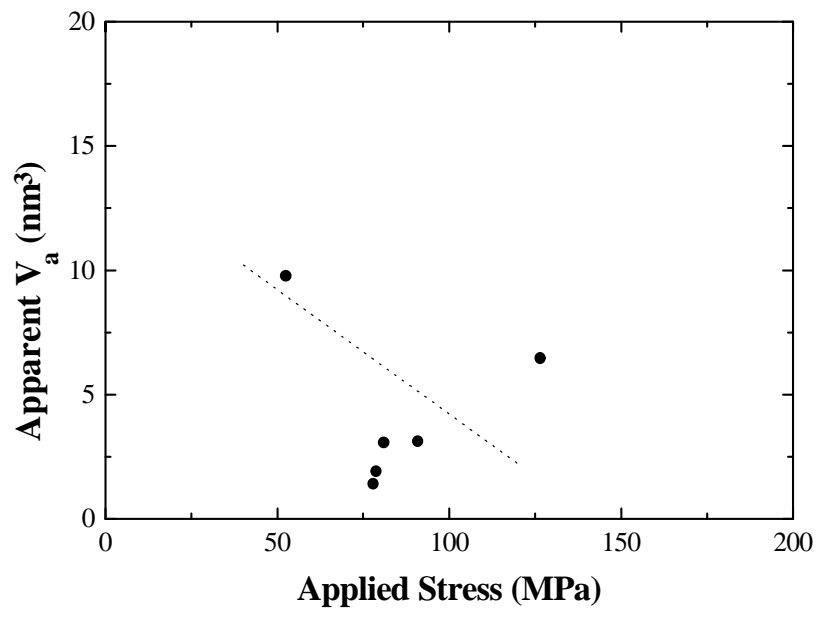

Figure 6. Apparent activation volume estimated from stress relaxation.

\section{Acknowledgements}

We would like to thank Mike Williams at Morgan Matroc, Transducer Division, Southampton and the Engineering and Physical Science Research Council (Grant No GR/L90361) for supporting this work.

\section{References}

Calderon Moreno J M, Guiu F, Meredith M, Reece M J, Alford N M and Penn S J 1999 J. Eur. Ceram. Soc. 191321

Esaklul K A, Gerberich W W and Koepke B G $1980 \mathrm{~J}$. Am. Ceram. Soc. 6325

Guiu F and Pratt P L 1964 Phys. Status Solidi 6111

MacEwen S R, Kupcis O A and Ramaswami B 1969 Scr. Metall. 3441 